\title{
Relationship Between Arterial-Alveolar 0xygen Gradient, Mortality and Admission to Intensive Care Unit in Severe Covid-19 Related Pneumonia: A Pilot Study
}

\author{
Gabrielli M*, Esperide A, Valletta F, Giancristofaro F, Santoro M, Santarelli L, and Franceschi F \\ Fondazione Policlinico Universitario a Gemelli IRCCS, Università Cattolica del Sacro Cuore, Department of Emergency, Rome, \\ Italy
}

*Corresponding author: Maurizio Gabrielli, Fondazione Policlinico Universitario A Gemelli IRCCS, Università Cattolica del Sacro Cuore Department of Emergency MedicineLargo Gemelli 8, 00168, Rome, Italy

\section{ARTICLE INFO}

Received: 半 September 28, 2020

Published: 慧 October 08, 2020

Citation: Gabrielli M, Esperide A, Valletta F, Giancristofaro F, Santoro M, Santarelli L, Franceschi F. Relationship Between Arterial-Alveolar Oxygen Gradient, Mortality and Admission to Intensive Care Unit in Severe Covid-19 Related Pneumonia: A Pilot Study. Biomed J Sci \& Tech Res 31(1)-2020. BJSTR. MS.ID.005039.

Keywords: SARS-CoV-2; COVID-19; Pneumonia; p02/FO2; A-a 02 Gradient

Abbreviations: ICU: Intensive Care Unit; ED: Emergency Department; ABG: Arterial Blood Gas; CAP: Community Acquired Pneumonia; ARDS: Acute Respiratory Distress Syndrome; MODS: Multiple Organ Dysfunction Syndrome

\begin{abstract}
Background: Recent studies evaluated, among demographics, clinical symptoms and vital signs, comorbidities, laboratory and imaging findings, the risk factors for mortality or admission to Intensive Care Unit (ICU) of patients with COVID-19 pneumonia. Alveolararterial oxygen $\left(\mathrm{A}-\mathrm{a} \mathrm{O}_{2}\right)$ gradient resulted as a good predictor of mortality in patients admitted for community acquired pneumonia (CAP). No data are available concerning the utility of A-a 02 gradient to stratify the risk of mortality or ICU admission in COVID-19 related pneumonia.
\end{abstract}

Methods: We retrospectively enrolled consecutive adult patients with pneumonia associated with laboratory-confirmed SARS-CoV2 infection, evaluated in our Emergency Department (ED) from 1 March to 1 May 2020. We considered only patients admitted to our hospital, finally classified as critical illness since early (within 24 hours) or later admission to ICU, and with availability of data from arterial blood gas analysis (ABG) performed on ambient air. $\mathrm{A}-\mathrm{a} \mathrm{O}_{2}$ gradient and $\mathrm{A}$-a $\mathrm{O} 2$ difference were calculated. Demographic variables, clinical symptoms, medical history, laboratory findings were also recorded for all patients included in the study.

Results: A total of 38 patients were finally enrolled (31 males, mean age of $65.2 \pm 12.9$ years).

Thirteen $(13,34 \%)$ died. No difference in mortality was observed between patients early admitted to ICU with respect to those transferred later ( $41 \%$ vs $31 \%$, p=ns).A-a 02 gradient, both with $\mathrm{PaO} 2, \mathrm{PaCO} 2, \mathrm{PaO} 2 / \mathrm{FiO} 2, \mathrm{pH}$, and lactate, did not significantly differ between survivors and non survivors. A-a 02 gradient, but not the other ABG findings, showed significantly higher values in the early ICU admission group versus patients with late clinical worsening.

Conclusion: In our preliminary study $\mathrm{A}-\mathrm{a} \mathrm{O} 2$ gradient, calculated at arrival in the ED, in patients with severe COVID-19 pneumonia, was able to predict early admission to ICU but not mortality.

\section{Introduction}

The Severe Acute Respiratory Syndrome Coronavirus 2 (SARSCoV-2) is the causative agent of the respiratory disease later named Coronavirus Disease 2019 (COVID-19), remaining a world public health emergency at present [1,2]. The disease is mild in most people, but in some, especially the elderly and those with comorbidities, it may progress to more severe pictures, often represented by 
pneumonia, until acute respiratory distress syndrome (ARDS) and multiple organ dysfunction syndrome (MODS)[1,2]. Recent retrospective studies on large Chinese population samples assessed the risks of mortality and the occurrence of critical illness in patients with COVID-19 [3-6].Several risk factors, with variable impact on prognosis across different trials, were identified among demographics (such as older age, male sex), symptoms (such as fever, dyspnea, haemoptysis, unconsciousness), number and type of comorbidities (the most important being arterial hypertension and malignancies), laboratory findings (neutrophil/lymphocites ratioNLR, lactate dehydrogenase - LDH, direct bilirubin, creatinine, transaminase, D-dimer, C reactive protein - CRP, procalcitonin, troponin, natriuretic peptides), abnormalities to chest imaging [36].

Combination of different risk factors into prognostic models led researchers to identify and validate some nomograms or calculation tools, apparently endowed with a good performance (area under the curve, AUC $>0.8$ in all trials) [3-6].Although pneumonia-associated hypoxemia is the main complication causing hospital admission in COVID-19 patients, none of these studies considered the impact of ABG parameters, and in particular of the alveolar-arterial gradient. In a really interesting editorial entitled "Basing Respiratory Management of COVID-19 on Physiological Principles", [7] Tobin underlined the importance of calculating the alveolar-arterial oxygen (A-a 02) gradient, since it is able to assess the pathophysiological basis of the hypoxemia more precisely than the commonly used ratio $\mathrm{PaO} 2 / \mathrm{FiO2}$. In fact, $\mathrm{PaO} 2 / \mathrm{FiO} 2$ may have minor accuracy, because it is influenced by changes in two parameters ( $\mathrm{PaO} 2$ and/or $\mathrm{FiO2}$ ) [7]. Consistent with this proposal, we decided to assess if the calculation of $\mathrm{A}-\mathrm{aO} 2$ upon arrival in the emergency room is a predictive factor of two principal outcomes in patients with COVID-19 pneumonia:

1) Mortality,

2) Early (within 24 hours) admission to Intensive Care Unit (ICU).

\section{Methods}

We retrospectively reviewed the medical records of consecutive patients with laboratory-confirmed infection by SARS-CoV2 admitted to the Gemelli Hospital of Rome, Italy, from 1 March to 1 May 2020, and finally classified as critical cases (admission to ICU).

Inclusion criteria were:

1) Age $>18$ years,

2) Presence at arrival of at least one symptom among fever $\left(\geq 38^{\circ} \mathrm{C}\right)$, cough, dyspnea,

3) Real-time reverse-transcription polymerase-chainreaction (RT-PCR) assay for nasal and pharyngeal swab specimens positive for SARS-CoV2,
4) Radiologic evidence of pneumonia (chest computed tomography or x-rays),

5) Hospitalization,

6) Availability of the following data: demographic variables, clinical symptoms, medical history, laboratory findings including arterial blood gas analysis (ABG), which should have been performed on room air (patients were excluded if the ABG was made on oxygen supplementation).

The $\mathrm{A}-\mathrm{a} \mathrm{O}_{2}$ gradient and the $\mathrm{A}-\mathrm{a} \mathrm{O}_{2}$ difference were calculated for each patient and expressed in mmHg. The calculation of gradient was done as follows: [150 - (1.25 x PaC02)] - Pa02. The $\mathrm{A}-\mathrm{a} \mathrm{O}_{2}$ difference was obtained by subtracting the expected $\mathrm{A}-\mathrm{a} \mathrm{O}_{2}$ to the measured $\mathrm{A}-\mathrm{a} 02$ gradient. The expected $\mathrm{A}-\mathrm{a} \mathrm{O}_{2}$ gradient was derived by the formula (Age/4) +4 .

The present study satisfies the Helsinki criteria.The statistical analysis was performed with SPSS 21.0 (SPSS, Florence, Italy). Continuous data were expressed as mean \pm 1 standard deviation and categoric variables as percentages. The normal distribution was determined by Kolmogorov-Smirnov test. Mean values of continuous variables were compared between the groups using Mann-Whitney U test. Student's t-test was used in the comparison of parameters showing normal distribution. The results were evaluated as $95 \%$ confidence interval and $p$ value $<0.05$, which was considered statistically significant.

\section{Results}

After full evaluation of 134 consecutive patients with COVID19-related pneumonia, 38 fulfilled all the requested inclusion criteria, then were included in the present study. Most were males $(31,82 \%)$ with a mean age of $65 \pm 13$ years. Seven (7) out of 38 $(18 \%)$ were active smokers, 20 (53\%) had arterial hypertension, $8(21 \%)$ diabetes mellitus, $3(8 \%)$ chronic obstructive pulmonary disease (COPD), 2 (5\%) history of heart failure, 7 (18\%) history of ischemic heart disease, 7 (18\%) previous or actual malignancies. Of note, about home treatment, 6 (17\%) patients were assuming angiotensin-converting enzyme inhibitors (ACE-i) and 6 (17\%) angiotensin II receptor blockers (ARB). The COVID-19 related symptoms at presentation were fever $(37,97 \%)$, dyspnea $(21$, $55 \%)$, cough $(17,45 \%)$, other symptoms (such as headache, chest pain, gastrointestinal symptoms: $6,16 \%$ ). A total of 13 out of 38 patients (34\%) died during their hospital stay. No difference in mortality was observed between the group of patients early admitted to ICU (within 24 hours of arrival at the hospital, 9/22, $41 \%$ ) with respect to those transferred later from other wards to ICU $(4 / 16,31 \%, p=n s)$.

The differences between the groups (survivors versus non survivors) concerning demographic variables, symptoms, medical history, anti-hypertensive treatments, and principal vital signs recorded upon arrival in the emergency room were summarized 
in (Table 1). Of note, older age and treatment with ACE-1/ARB were significantly associated with mortality. Instead, no significant differences emerged between groups about comorbidities, although arterial hypertension showed a higher prevalence in the group of non survivors.(Table 2)showed the distribution of the principal parameters deriving from the ABG assessed in ambient air upon arrival in the two groups. No significant difference was observed about oxygen saturation, $\mathrm{PaO} 2, \mathrm{PaCO} 2, \mathrm{PaO} 2 / \mathrm{FiO} 2, \mathrm{pH}$,
A-a 02 gradient, A-a 02 difference, and lactate level, although lactate showed a trend toward an higher concentration in non survivors ( $\mathrm{p}=0.055)$. A-a 02 gradient and $\mathrm{A}-\mathrm{a} 02$ difference were instead associated with the second outcome: both they showed significantly higher values in the early ICU admission group with respect to patients admitted in ICU after 24 hours (Table 3). The same thing did not happen for the other ABG parameters, including $\mathrm{PaO} 2 / \mathrm{FiO} 2$.

Table 1: Differences between survivors and non survivors about demographic variables, symptoms, medical history, anti-hypertensive treatments, and principal vital signs recorded at arrival. Results regarding other comorbidities such as diabetes mellitus, COPD, cardiovascular diseases, malignancies were not reported in the table since the low prevalence detected in our population.

\begin{tabular}{|c|c|c|c|}
\hline & Survivors (25) & Non survivors (13) & p \\
\hline Age (years) & $60.5 \pm 13.2$ & $74.1 \pm 5.7$ & 0.001 \\
\hline Sex (female) & $80 \%$ & $85 \%$ & $\mathrm{~ns}$ \\
\hline Arterial hypertension & $44 \%$ & $69 \%$ & $\mathrm{~ns}$ \\
\hline Fever & $100 \%$ & $92 \%$ & $\mathrm{~ns}$ \\
\hline Dyspnea & $56 \%$ & $54 \%$ & $\mathrm{~ns}$ \\
\hline Cough & $48 \%$ & $38 \%$ & 0.02 \\
\hline Systolic BP (mm Hg) & $135.1 \pm 17.8$ & $121.5 \pm 21.6$ & $\mathrm{~ns}$ \\
\hline Diastolic BP (mm Hg) & $83.2 \pm 11.2$ & $73.8 \pm 15.1$ & $\mathrm{~ns}$ \\
\hline Heart rate $>100$ bpm & $40 \%$ & $38 \%$ & $\mathrm{~ns}$ \\
\hline Respiratory rate $\geq 24$ breaths/min & $12 \%$ & $15 \%$ & 0.006 \\
\hline
\end{tabular}

Table 2: Differences between survivors and non survivors about the principal parameters of arterial blood gas analysis assessed in ambient air upon arrival.

\begin{tabular}{|c|c|c|c|}
\hline & Survivors (25) & Non survivors (13) & p \\
\hline Oxygen saturation (\%) & $86.5 \pm 14.2$ & $87.7 \pm 11.4$ & $\mathrm{~ns}$ \\
\hline PaO2 (mmHg) & $58.4 \pm 14.3$ & $59.1 \pm 22.8$ & $\mathrm{~ns}$ \\
\hline PaCO2 (mmHg) & $32.5 \pm 4.5$ & $34.0 \pm 7.9$ & $\mathrm{~ns}$ \\
\hline PaO2/FiO2 & $277.9 \pm 68.3$ & $273.8 \pm 98.5$ & $\mathrm{~ns}$ \\
\hline $\mathrm{pH}$ & $7.41 \pm 0.05$ & $7.41 \pm 0.06$ & $\mathrm{~ns}$ \\
\hline AaDO2 (mmHg) & $50.6 \pm 15.3$ & $49.1 \pm 21.8$ & $\mathrm{~ns}$ \\
\hline AaDO2 augmentation (mmHg) & $31.6 \pm 15$ & $27.0 \pm 20.6$ & $\mathrm{~ns}$ \\
\hline Lactate level (mmol/L) & $1.2 \pm 0.5$ & $1.8 \pm 1.3$ & $\mathrm{~ns}$ \\
\hline
\end{tabular}

Table 3: Differences between the group of patients early admitted to ICU (within 24 hours since arrival at emergency room) and the group of those admitted later concerning the principal parameters of arterial blood gas analysis assessed in ambient air upon arrival in the ED.

\begin{tabular}{|c|c|c|c|}
\hline & Early ICU admission (16) & Late ICU admission (22) & p \\
\hline Oxygen saturation (\%) & $86.7 \pm 7.6$ & $87.1 \pm 16.3$ & $\mathrm{~ns}$ \\
\hline PaO2 (mmHg) & $53.3 \pm 16.1$ & $62.6 \pm 17.6$ & $\mathrm{~ns}$ \\
\hline PaCO2 (mmHg) & $31.8 \pm 4.4$ & $33.9 \pm 6.6$ & $\mathrm{~ns}$ \\
\hline Pa02/FiO2 & $247.3 \pm 62.4$ & $297.7 \pm 83.7$ & $\mathrm{~ns}$ \\
\hline $\mathrm{pH}$ & $7.43 \pm 0.07$ & $7.40 \pm 0.04$ & 0.016 \\
\hline AaD02 (mmHg) & $57.6 \pm 16.4$ & $44.7 \pm 16.6$ & $\mathrm{~ns}$ \\
\hline Lactate level (mmol/L) & $36.9 \pm 16.3$ & $25.0 \pm 15.9$ & 0.023 \\
\hline
\end{tabular}


None of the principal laboratory tests (blood count, coagulation, renal and liver function, LDH, CRP, procalcitonin), dosed at arrival, showed a significant correlation either with patients' survival or with early ICU admission (data not shown). CRP tended to be higher in the non-survivor's group $(200.1 \pm 100.8 \mathrm{mg} / \mathrm{L})$ with respect to survivors $(126.3 \pm 86.1 \mathrm{mg} / \mathrm{L}, \mathrm{p}=0.07)$.

\section{Discussion}

The AaDO2 is the measure of the difference between the oxygen concentration in the alveoli and in the arterial system [7]. It has a fundamental clinical utility as it helps to evaluate if hypoxemia is due to dysfunction of the alveolar-capillary unit ("lung" failure, increased A-a 02 gradient) or to a "pump" failure (normal A-a 02 gradient). Hypoxemia with COVID-19 is generally associated with an increased alveolar-to-arterial oxygen gradient, due to either ventilation-perfusion mismatch or intrapulmonary shunt, linked to a (variable) inflammatory involvement of pulmonary parenchyma [8]. It could be hypothesized that, in patients with COVID-19 pneumonia, the higher the gradient, the more severe the lung inflammatory damage and then higher the risk of death. This hypothesis has been already proven in patients with community acquired pneumonia (CAP) $[9,10]$. In a recently published study, Avci et al. [10] demonstrated that A-a 02 gradient and difference were potent predictors of 30-day mortality in patients with CAP. The AaDO2 and AaO2 difference were even more potent than inflammatory markers as CRP or score as PSI or CURB-65.Data on prognostic utility of A-a 02 gradient in patients with COVID-19 related pneumonia are scarce at present.

In a small recent study performed at an Emergency Department in Naples, A-a 02 gradient and A-a 02 difference, both with oxygen saturation, $\mathrm{PaO} 2, \mathrm{PaO} 2 / \mathrm{FiO} 2$, and lactate, resulted good predictors of ICU admission in patients with COVID-19 [11]. In our study, A-a 02 gradient and A-a 02 difference measured at arrival (but not oxygen saturation, paO2, $\mathrm{PaO} 2 / \mathrm{FiO} 2$, and lactate) were able to stratify patients that were admitted to ICU within 24 hours. In addition, our findings suggest that neither $\mathrm{A}-\mathrm{a} 02$ gradient and its difference nor the other $A B G$ parameters are able to identify patients with COVID-19 pneumonia at risk of death. Once patients in which ABG at arrival was performed during oxygen supplementation were excluded in order to avoid any possible bias, our sample was rather small (only 38 out of the initially assessed 134). This is undoubtedly one of the major limitations to our study, another being its retrospective design.Anyway, our population of severe COVID-19 pneumonia showed characteristics that were similar to larger samples reported in the literature: predominance of male sex; mortality rate similar to that reported by the literature for the severe/critical cases; age as the most important factor associated with mortality, both with the problem of arterial hypertension and its treatment with ACE-i/ARB, which have been postulated to play a role in the pathogenesis of SARS-CoV2 infection [12-14]
Interestingly, the group with poor prognosis showed at arrival significantly lower values of systolic and a trend toward lower diastolic blood pressure and higher lactate and CRP levels, as if these patients were heading towards ahemodynamic impairment. These abnormalities look like first signs of an incipient 'viral sepsis' or 'cytokines storm', typical of more severe and often mortal COVID-19 cases $[15,16]$. In conclusion, our preliminary study represents a hypothesis-generating paper. The alveolar-to-arterial gradient and its difference seemed useful to well stratify patients at risk of early precipitation of respiratory failure but were not related to mortality. Further, possibly prospective, studies on a larger population are needed to confirm the present findings.

\section{Conflicts of Interest}

We have no conflict of interests to disclose and the manuscript has been read and approved by all named authors.

\section{Acknowledgement}

\section{Below are listed all members of the GEMELLI AGAINST COVID Study Group}

Abbate Valeria, Acampora Nicola, Addolorato Giovanni, Agostini Fabiana, Ainora Maria Elena, Akacha Karim, Amato Elena, Andreani Francesca, Andriollo Gloria, Annetta Maria Giuseppina, Annicchiarico Brigida Eleonora, Antonelli Mariangela, Antonucci Gabriele, Anzellotti Gian Marco, Armuzzi Alessandro, Baldi Fabiana, Barattuccillaria, Barillaro Christian, Barone Fabiana, Bellantone Rocco Domenico Alfonso, Bellieni Andrea, Bello Giuseppe, Benicchi Andrea, Benvenuto Francesca, BerardiniLudovica, Berloco Filippo, Bernabei Roberto, Bianchi Antonio, Biasucci Daniele Guerino, Biasucci Luigi Marzio, Bibbò Stefano, Bini Alessandra, Bisanti Alessandra, Biscetti Federico, Bocci Maria Grazia, Bonadia Nicola, Bongiovanni Filippo, Borghetti Alberto, Bosco Giulia, Bosello Silvia, Bove Vincenzo, Bramato Giulia, Brandi Vincenzo, Bruni Teresa, Bruno Carmine, Bruno Dario, Bungaro Maria Chiara, Buonomo Alessandro, Burzo Livia, Calabrese Angelo, Calvello Maria Rosaria, Cambieri Andrea, Cambise Chiara, Cammà Giulia, Candelli Marcello, Canistro Gennaro, Cantanale Antonello, Capalbo Gennaro, Capaldi Lorenzo, Capone Emanuele, Capristo Esmeralda, Carbone Luigi, Cardone Silvia, Carelli Simone, Carfi Angelo, Carnicelli Annamaria, Caruso Cristiano, Casciaro Francesco Antonio, Catalano Lucio, Cauda Roberto, Cecchini Andrea Leonardo, Cerrito Lucia, Cesarano Melania, Chiarito Annalisa, CianciRossella, Cicetti Marta, Cicchinelli Sara, Ciccullo Arturo, Ciciarello Francesca, Cingolani Antonella, Cipriani Maria Camilla, Consalvo Maria Ludovica, Coppola Gaetano, Corbo Giuseppe Maria, Corsello Andrea, Costante Federico, Costanzi Matteo, Covino Marcello, Crupi Davide, Cutuli Salvatore Lucio, D’Addio Stefano, D’Alessandro Alessia, D’alfonso Maria Elena, D’Angelo Emanuela, D'Aversa Francesca, Damiano Fernando, De Berardinis Gian Maria, De Cunzo Tommaso, de Gaetano DonatiKatleen, De Luca Giulio, De Matteis Giuseppe, 
De Pascale Gennaro, De Santis Paolo, De Siena Martina, De Vito Francesco, Del Gatto Valeria, Del Giacomo Paola, Del Zompo Fabio, Dell'Anna Antonio Maria, Della Polla Davide, Di Gialleonardo Luca, Di Giambenedetto Simona, Di Luca Roberta, Di Maurizio Luca, Di Muro Mariangela, Dusina Alex, Eleuteri Davide, Esperide Alessandra, Facheci Daniele, Faliero Domenico, FalsiroliCinzia, Fantoni Massimo, Fedele Annalaura, Feliciani Daniela, Ferrante Cristina, Ferrone Giuliano, Festa Rossano, Fiore Maria Chiara Flex Andrea, Forte Evelina, Franceschi Francesco, Francesconi Alessandra, Franza Laura, Funaro Barbara, Fuorlo Mariella, Fusco Domenico, Gabrielli Maurizio, Gaetani Eleonora, Galletta Claudia, Gallo Antonella, Gambassi Giovanni, Garcovich Matteo, Gasbarrini Antonio, Gasparrini Irene, Gelli Silvia, Giampietro Antonella, Gigante Laura, Giuliano Gabriele, Giuliano Giorgia, Giupponi Bianca, Gremese Elisa, Grieco Domenico Luca, Guerrera Manuel, Guglielmi Valeria, Guidone Caterina, Gullì Antonio, Iaconelli Amerigo, Iafrati Aurora, Ianiro Gianluca, Iaquinta Angela, Impagnatiello Michele, Inchingolo Riccardo, IntiniEnrica, Iorio Raffaele, IzziImmacolata Maria, Jovanovic Tamara, Kadhim Cristina, La Macchia Rosa, La Milia Daniele Ignazio, Landi Francesco, Landi Giovanni, Landi Rosario, Landolfi Raffaele, Leo Massimo, Leone Paolo Maria, Levantesi Laura, Liguori Antonio, Liperoti Rosa, Lizzio Marco Maria, Lo Monaco Maria Rita, Locantore Pietro, Lombardi Francesco, Lombardi Gianmarco, Lopetuso Loris, Loria Valentina, Losito Angela Raffaella, Lucia Mothanje Barbara Patricia, Macagno Francesco, Macerola Noemi, Maggi Giampaolo, Maiuro Giuseppe, Mancarella Francesco, Mangiola Francesca, Manno Alberto, Marchesini Debora, Maresca Gian Marco, Marrone Giuseppe, Martis Ilaria, Martone Anna Maria, Marzetti Emanuele, Mattana Chiara, Matteo Maria Valeria, Maviglia Riccardo, Mazzarella Ada, Memoli Carmen, Miele Luca, Migneco Alessio, Mignini Irene, Milani Alessandro, Milardi Domenico, Montalto Massimo, Montemurro Giuliano, Monti Flavia, Montini Luca, Morena Tony Christian, MorraVincenzina, Moschese Davide, Murace Celeste Ambra, Murdolo Martina, Murri Rita, Napoli Marco, Nardella Elisabetta, NatalelloGerlando, Natalini Daniele, Navarra Simone Maria, Nesci Antonio, Nicoletti Alberto, Nicoletti Rocco, Nicoletti Tommaso Filippo, Nicolò Rebecca, Nicoletti Rocco, NicolottiNicola, Nista Enrico Celestino, Nuzzo Eugenia, Oggiano Marco, Ojetti Veronica, Pagano Francesco Cosimo, Paiano Gianfranco, Pais Cristina, Paolillo Federico, Pallavicini Federico, Palombo Andrea, Papa Alfredo, Papanice Domenico, Papparella Luigi Giovanni, Paratore Mattia, Parrinello Giuseppe, Pasciuto Giuliana, PasculliPierpaolo, Pecorini Giovanni, Perniola Simone, Pero Erika, Petricca Luca, Petrucci Martina, Picarelli Chiara, Piccioni Andrea, Piccolo Annalisa, PiervincenziEdoardo, Pignataro Giulia, Pignataro Raffaele, Pintaudi Gabriele, Pisapia Luca, Pizzoferrato Marco, Pizzolante Fabrizio, Pola Roberto, Policola Caterina, Pompili Maurizio, Pontecorvi Flavia, Pontecorvi Valerio, Ponziani Francesca, Popolla Valentina, PorcedduEnrica, Porfidia Angelo, Porro Lucia Maria, Potenza Annalisa, Pozzana Francesca, Privitera
Giuseppe, Pugliese Daniela, Pulcini Gabriele, Racco Simona, Raffaelli Francesca, Ramunno Vittoria, Rapaccini Gian Ludovico, Richeldi Luca, Rinninella Emanuele, Rocchi Sara, Romanò Bruno, Romano Stefano, Rosa Federico, Rossi Laura, Rossi Raimondo, Rossini Enrica, Rota Elisabetta, Rovedi Fabiana, Rubino Carlotta, Rumi Gabriele, Russo Andrea, Russo Andrea, Sabia Luca, Salerno Andrea, Salini Sara, Salvatore Lucia, Samori Dehara, Sandroni Claudio, Sanguinetti Maurizio, Santarelli Luca, Santini Paolo, Santolamazza Danilo, Santoliquido Angelo, Santopaolo Francesco, Santoro Michele Cosimo, Sardeo Francesco, Sarnari Caterina, Saviano Angela, Saviano Luisa, Scaldaferri Franco, Scarascia Roberta, Schepis Tommaso, Schiavello Francesca, Scoppettuolo Giancarlo, Sedda Davide, Sessa Flaminio, Sestito Luisa, Settanni Carlo, Siciliano Matteo, Siciliano Valentina, SicuranzaRossella, SimeoniBenedetta, Simonetti Jacopo, Smargiassi Andrea, Soave Paolo Maurizio, Sonnino Chiara, Staiti Domenico, Stella Claudia, Stella Leonardo, Stival Eleonora, Taddei Eleonora, TalericoRossella, Tamburello Elio, TamburriniEnrica, Tanzarella Eloisa Sofia, Tarascio Elena, Tarli Claudia, Tersali Alessandra, Tilli Pietro, Timpano Jacopo, Torelli Enrico, Torrini Flavia, Tosato Matteo, Tosoni Alberto, Tricoli Luca, Tritto Marcello, Tumbarello Mario, Tummolo Anita Maria, Vallecoccia Maria Sole, Valletta Federico, Varone Francesco, Vassalli Francesco, Ventura Giulio, Verardi Lucrezia, Vetrone Lorenzo, Vetrugno Giuseppe, Visconti Elena, Visconti Felicia, Viviani Andrea, Zaccaria Raffaella, Zaccone Carmelina, Zelano Lorenzo, Zileri Dal Verme Lorenzo, Zuccalà Giuseppe.

\section{References}

1. Pascarella G, Strumia A, Piliego C, Bruno F, Del Buono R, et al. (2020) COVID-19 diagnosis and management: A comprehensive review. J Intern Med 288: 192-206.

2. Guan WJ, Ni ZY, Hu Y, Liang WH, Ou CQ, et al. (2020) Clinical Characteristics of Coronavirus Disease 2019 in China. N Engl J Med 382(18): 1708-1720.

3. Zheng Z, Peng F, Xu B, Zhao J, Liu H, et al. (2020) Risk factors of critical \& mortal COVID-19 cases: A systematic literature review and metaanalysis. J Infect 81: e16-e25.

4. Liang W, Liang H, Ou L, Chen B, Chen A, et al. (2020) Development and Validation of a Clinical Risk Score to Predict the Occurrence of Critical Illness in Hospitalized Patients With COVID-19. JAMA Intern Med 180(8): 1081-1089.

5. Dong YM, Sun J, Li YX, Chen Q, Liu QQ, et al. (2020) Development and Validation of a Nomogram for Assessing Survival in Patients with COVID-19 Pneumonia. Clin Infect Dis, ciaa963.

6. Zhang S, Guo M, Duan L, Wu F, Hu G, et al. (2020) Development and validation of a risk factor-based system to predict short-term survival in adult hospitalized patients with COVID-19: A multicenter, retrospective, cohort study. Crit Care 24(1): 438.

7. Tobin MJ (2020) Basing Respiratory Management of COVID-19 on Physiological Principles. Am J Respir Crit Care Med 201(11): 1319-1320.

8. Tobin MJ, Laghi F, Jubran A (2012) Ventilatory failure, ventilator support, and ventilator weaning. Compr Physiol 2: 2871-2921.

9. Moammar MQ, Azam HM, Blamoun AI, Rashid AO, Ismail M, et al. (2008) Alveolar-arterial oxygen gradient, pneumonia severity index and outcomes in patients hospitalized with community acquired pneumonia. Clin Exp Pharmacol Physiol 35(9): 1032-1037. 
10. Avci S, Perincek G (2020) The alveolar-arterial gradient, pneumonia severity scores and inflammatory markers to predict 30-day mortality in pneumonia. Am J Emerg Med 38(9): 1796-1801.

11. Carlino MV, Valenti N, Cesaro F, Costanzo A, Cristiano G, et al. (2020) Predictors of Intensive Care Unit admission in patients with coronavirus disease 2019 (COVID-19). Monaldi Arch Chest Dis 90(3).

12. Grasselli G, Zangrillo A, Zanella A, Antonelli M, Cabrini L, et al. (2020) Baseline characteristics and outcomes of 1591 patients infected with SARS-CoV-2 admitted to ICUs of the Lombardy region, Italy. JAMA 323(16): 1574-1581.

13. Imam Z, Odish F, Gill I, O'Connor D, Armstrong J, et al. (2020) Older age and comorbidity are independent mortality predictors in a large cohort

\section{ISSN: 2574-1241}

DOI: 10.26717/BJSTR.2020.31.005039

Gabrielli M. Biomed J Sci \& Tech Res

(C) This work is licensed under Creative

Submission Link: https://biomedres.us/submit-manuscript.php of 1305 COVID-19 patients in Michigan, United States. J Intern Med 288(4): 469-476.

14. Rico Mesa JS, White A, Anderson AS (2020) Outcomes in Patients with COVID-19 Infection Taking ACEI/ARB. Curr Cardiol Rep 22(5): 31.

15. Juneja D, Singh O, Dang R (2011) Admission hyperlactatemia: causes, incidence, and impact on outcome of patients admitted in a general medical intensive care unit. J Crit Care 26(3): 316-320.

16. Zhang C, Wu Z, Li JW, Zhao H, Wang GQ (2020) Cytokine release syndrome in severe COVID-19: interleukin-6 receptor antagonist tocilizumab may be the key to reduce mortality. Int J Antimicrob Agents. 55(5): 105954

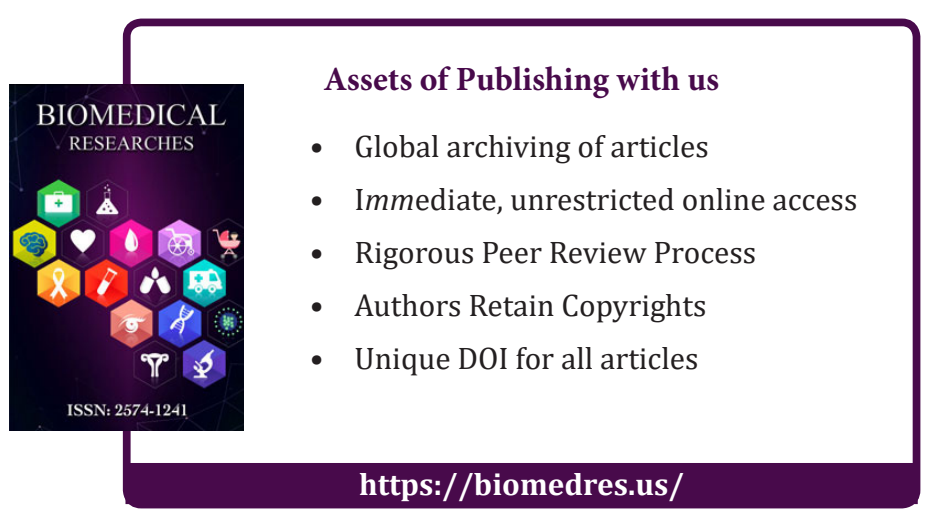

